\title{
How to Enable Collaboration in Open Government Data Ecosystems: A Public Platform Provider's Per- spective
}

\section{Johan Linåker and Per Runeson}

Dept. of Computer Science, Lund University, Lund (SWE), johan.linaker@cs.lth.se, per.runeson@cs.lth.se

Abstract: Open Government Data (OGD) is an important driver for open innovation among public entities. However, extant research highlights a need for improved feedback loops, collaboration, and a more demand-driven publication of OGD. In this study, we explore how public platform providers can address this issue by enabling collaboration within OGD ecosystems, both in terms of the OGD, and any related Open Source Software (OSS) and standards. We conducted an exploratory multiple-case study of four OGD ecosystems with diverse characteristics, using a qualitative research approach. Based on the cases, we present a conceptual model that highlights different attributes of OGD ecosystems that may help public entities in designing and orchestrating new or existing OGD ecosystems. We conclude that enabling collaboration in an OGD ecosystem is a complex exercise yet believe that it offers ways for public entities in how they can leverage open innovation to address their goals and directives.

Keywords: Open government data, Open data, Open Source Software, Public sector, Data ecosystem

Acknowledgement: The research was funded through the JobTech Research Project, a collaboration between Lund University and SPES. The authors would like to thank the interviewees for their time and honesty, as well as the reviewers to this study, and the conference paper on which this study is based.

\section{Introduction}

Rich and high-quality data have over time become a critical asset for software organizations as a driver for innovation and input to solutions including artificial intelligence (Munappy et al. 2019; Gao and Janssen 2020). One way of increasing access and availability of such data is to share it as Open Data (Attard et al. 2015) and collaborate on its collection and maintenance as commonly done with Open Source Software (OSS) (Munir, Wnuk, and Runeson 2016). Such sharing of data is less common within the software industry (Runeson 2019), but more so among public entities (Safarov, Meijer, and Grimmelikhuijsen 2017). In the latter case, we refer to the openly shared data as Open 
Government Data (OGD) (Attard et al. 2015). More specifically, we consider OGD as “...governmentrelated data that is made open to the public" (Attard et al. 2015) and thereby a subset of Open Data.

To enable the potential innovation output from OGD (Lakomaa and Kallberg 2013; Janssen, Charalabidis, and Zuiderwijk 2012), public entities along with cross-sector organizations and citizens may form OGD ecosystems. These function as value networks through which the OGD is enriched and used as input to new or improved products and services (Lindman, Kinnari, and Rossi 2015; Zuiderwijk, Janssen, and Davis 2014). The interaction and collaboration in these ecosystems are however often quite limited (Najafabadi and Luna-Reyes 2017; Immonen, Palviainen, and Ovaska 2014; Dawes, Vidiasova, and Parkhimovich 2016), even though it is highlighted as a need (Sieber and Johnson 2015; Rudmark and Hjalmarsson-Jordanius 2019) and implicit characteristics of a data ecosystem (Oliveira, Lima, and Lóscio 2019).

Considering OSS ecosystems (commonly referred to as communities) (Franco-Bedoya et al. 2017), open collaboration on the underpinning OSS projects is common practice. By working together in an open, transparent, and egalitarian manner (Feller, Fitzgerald, and others 2002), new functionality is continuously asserted, discussed, and implemented (Ernst and Murphy 2012), thereby accelerating the innovation and development beyond what any single actor within the ecosystem could perform alone (Munir, Wnuk, and Runeson 2016).

OGD ecosystems may, potentially, have an opportunity to improve the innovation output and enable further value creation by adopting such practices (Linåker and Runeson 2020b). Also, considering open data ecosystems, such as OpenStreetMap ${ }^{1}$ and Wikidata ${ }^{2}$, there may also be a potential for catalyzing both the sharing and adoption of OGD by extending collaboration to include open development of boundary resources (Dal Bianco et al. 2014) such as related standards and software as OSS (Zuiderwijk, Janssen, and Davis 2014; Immonen, Palviainen, and Ovaska 2014; Rudmark 2020). As we consider current definitions of data ecosystems (Oliveira, Lima, and Lóscio 2019) (including related concepts (Susha et al. 2020)) lacking in terms of these characteristics, we define a data ecosystem, adapted from three sources (Zuiderwijk, Janssen, and Davis 2014; Jansen 2020; Oliveira, Lima, and Lóscio 2019), as:

a networked community of actors (organizations and individuals), which base their relations to each other on a common interest (Zuiderwijk, Janssen, and Davis 2014), supported by an underpinning technological platform (Jansen 2020) that enables actors to process data (for example, find, archive, publish, consume, or reuse) as well as to foster innovation, create value, or support new businesses (Oliveira, Lima, and Lóscio 2019). Actors collaborate on the data and boundary resources (for example, software and standards), through the exchange of information, resources, and artifacts (Jansen 2020).

For OGD ecosystems specifically, the provider of the technological platform is constituted by a public entity (or part of one). Further, we consider any data as OGD, which is published by the public entity constituting the platform provider, whether it is produced by the public entity itself,

\footnotetext{
https://www.openstreetmap.org/

https://www.wikidata.org/
} 
or collected from or shared by an actor within the ecosystem. These actors may, for example, include public entities, firms, non-profit organizations, research institutions, and citizens.

Existing research has mainly focused on the collaborative practices used in OSS ecosystems (Alves, Oliveira, and Jansen 2018; Jansen 2020), and has in terms of OGD ecosystems (Attard et al. 2015) (and similar concepts (Susha et al. 2020)) been limited, both regarding collaboration on OGD or any related software or standard, even though identified as a need (Sieber and Johnson 2015; Oliveira, Lima, and Lóscio 2019; Rudmark and Hjalmarsson-Jordanius 2019). The research goal of this study is therefore to explore how public entities in the role of platform providers can enable collaboration within their OGD ecosystems, both in terms of the OGD published on the underpinning platform, as well as any related boundary resources in the form of OSS and standards.

We take the perspective of the public entity constituting the platform provider, as these manage the platform and the ecosystem around it. The platform provider lays out the development direction of the platform, decides what is shared, and establishes the governance structure (Baars and Jansen 2012), i.e., rules and processes for how the ecosystem can influence and collaborate on the development and any shared resources (for example, data, software, or standards). We find this as an interesting perspective as trust towards the platform provider is pivotal to enable the collaboration and growth of an ecosystem (Susha et al. 2020; Coyle, Diepeveen, and Wdowin 2020).

To address the goal of this study, we conducted a multiple-case study (Runeson et al. 2012) of four OGD ecosystems in which OSS, standards, and related collaborative practices are adopted, aiming to foster collaboration and increase the adoption of OGD. This is an extension of our previous work (Linåker and Runeson 2020a) where we reported on findings related to two of the four cases presented in this paper. The four ecosystems are governed by public entities in the role of platform providers and focus on OGD related to the labor market in Sweden, public transport in Sweden, and the region of Helsinki in Finland, as well as the City of Chicago, Illinois, USA. Based on our findings we synthesize and present a conceptual model that describes how OGD ecosystems may vary in terms of different aspects, for example including, platform ownership and scope, as well as sharing and collaboration practices. For each aspect, we provide recommendations based on the explored cases that, along with the conceptual model, may help public entities in designing and orchestrating new or existing OGD ecosystems with the ambition to enable collaboration with and within the ecosystems.

The rest of this paper is structured as follows. In section 2 we provide a background on software and OGD ecosystems and their governance structures. In section 3 we present our research design, followed by the descriptive results from our case studies in section 4 . In section 5 , we present and discuss our synthesis from the case studies, in the form of a conceptual model along with a number of recommendations. Finally, we summarize and conclude the paper in section 6.

\section{Background}

The frame of our analysis and discussion of the studied cases is set by literature on software and data ecosystems, and particularly their organizational and governance structures, as outlined below. 


\subsection{Software and Data Ecosystems}

Stemming from the literature on business and digital ecosystems (Jansen, Finkelstein, and Brinkkemper 2009; Iansiti and Levien 2004; Oliveira, Lima, and Lóscio 2019), software and data ecosystems share a common background, yet they differentiate in many ways.

Software Ecosystems (Alves, Oliveira, and Jansen 2018; Jansen 2020) offer a lens for analyzing how networked communities of organizations collaborate around their common interest in a central software technology (Mhamdia 2013). The central software technology, commonly referred to as a platform (Jansen, Finkelstein, and Brinkkemper 2009), can be viewed as a "foundation technology or set of components used beyond a single firm and that brings multiple parties together for a common purpose or to solve a common problem" (Gawer and Cusumano 2002). The platform provider enables the ecosystem to use and extend the functionalities of the underpinning platform by providing boundary resources, i.e., "the software tools and regulations that serve as the interface for the arm's-length relationship between the platform owner and the application developer." (Ghazawneh and Henfridsson 2013) Such resources can be of both technical (for example, API:s, developer tools, and libraries) and social character (for example, documentation, forums, and hackathons) (Dal Bianco et al. 2014).

A Data Ecosystem is defined by Oliveira et al. (Oliveira, Lima, and Lóscio 2019) as a "socio-technical complex network in which actors interact and collaborate with each other to find, archive, publish, consume, or reuse data as well as to foster innovation, create value, and support new businesses. When comparing the definitions of software and data ecosystems (Jansen, Finkelstein, and Brinkkemper 2009; Oliveira, Lima, and Lóscio 2019), similar characteristics can be recognized, for example, in terms of actors, their relationships, as well as use, sharing, and collaboration of common resources (Oliveira, Lima, and Lóscio 2019). One difference however is that this definition of a data ecosystem lacks the mentioning of an explicit underpinning technological platform and any associated boundary resources. This observation further adds to other definitions of data ecosystems surveyed by Oliveira et al. (Oliveira, Lima, and Lóscio 2019).

Instead of software platforms, the focus in data ecosystems is rather on the flow of data between the actors in the ecosystem in the form of a value-chain (Lindman, Kinnari, and Rossi 2015). A general distinction between the roles in such value-chains can be made between data providers and data users (Zuiderwijk, Janssen, and Davis 2014). The roles can be further refined into data providers, service providers, data brokers, application developers, application users, and infrastructure and tool providers (Immonen, Palviainen, and Ovaska 2014; Kitsios, Papachristos, and Kamariotou 2017). The data provider is usually constituted by a public-sector organization (Oliveira, Lima, and Lóscio 2019) as data sharing from private actors is not as widespread (Runeson 2019). Services or functions needed include an infrastructure to share the data (preferably from multiple providers), documentation, tools for application developers, help in finding use-cases, as well as the possibility to discuss, provide feedback and make requests (Immonen, Palviainen, and Ovaska 2014; Zuiderwijk, Janssen, and Davis 2014; Dawes, Vidiasova, and Parkhimovich 2016). Several researchers observe the need for improved feedback-loops, collaboration, and a more demand-driven publication of OGD (Rudmark and Hjalmarsson-Jordanius 2019; Zuiderwijk, Janssen, and Davis 2014; Dawes, Vidiasova, and Parkhimovich 2016; Najafabadi and Luna-Reyes 2017).

In this study, we introduce an adapted definition of a data ecosystem (see section 1) where we explicitly highlight the presence of an underpinning technological platform as well as associated 
boundary resources. Considering the definition of a platform by Gawer and Cusumano (Gawer and Cusumano 2002), we consider the platform as the technological mean that in part or full, enables the ecosystem's actors to process data (for example, find, archive, publish, consume, or reuse) as well as to foster innovation, create value, or support new businesses, inspired by Oliveira et al. (Oliveira, Lima, and Lóscio 2019). With the definition, we also look specifically at Open Government Data (OGD) ecosystems, i.e., data ecosystems with a public actor (or part of one) providing a platform for sharing OGD (Attard et al. 2015).

\subsection{Ecosystem Organizational and Governance Structures}

OGD ecosystems are typically centered around "government organizations as central actors, taking the initiative within networked systems organized to achieve specific goals related to innovation and good government" (Harrison, Pardo, and Cook 2012). Oliveira et al. (Oliveira, Lima, and Lóscio 2019) propose a classification of ecosystems' organizational structures, two of which highlight the position of the platform provider, either as an orchestrator and main data provider of the ecosystem, or more limited as an intermediary between data producers and users of the ecosystem.

Considering software ecosystems specifically with the definition by Jansen et al. (Jansen, Finkelstein, and Brinkkemper 2009), these structures blend and overlap. Dal Bianco et al. (Dal Bianco et al. 2014) mainly differentiate between two organizational structures; keystone-centric ecosystems where the platform is controlled by a central independent organization, and consortium-based ecosystems where the central organization is created and co-owned by the ecosystem's members.

Looking further, three types of roles are commonly referred to (Jansen 2020; Iansiti and Levien 2004). The first role is that of the platform provider who is the owner and supplier of the platform and thereby also usually the orchestrator of the ecosystem. As an orchestrator, the platform provider also decides on the governance model for the ecosystem, meaning how it maintains control and decides on the direction, but also on the governance structure, meaning "the distribution of rights and responsibilities among the [ecosystem's members], and the rules and protocols that need to be followed to make decisions regarding the [ecosystem]" (Alves, Oliveira, and Jansen 2018).

Keystone and niche players are two other roles within an ecosystem. A keystone is an actor who nurtures a symbiotic relationship with the ecosystem and its other actors, looking to actively improve its health (Jansen 2014). Usually, they have a close connection with the platform provider, who also may be referred to as a keystone if it has similar symbiotic intents. Niche players are actors focused more on a specific niche of the market, or use-case, and is primarily a user of the resources provided by the ecosystem (Iansiti and Levien 2004).

For OSS ecosystems, the platform provider can be the owner of the OSS project, usually either a software vendor or the ecosystem of actors directly or via a proxy organization (for example, a foundation) (Riehle 2012). Governance, however, does not have to be aligned with the ownership. In more autocratic ecosystems, it can be centered around a vendor or an individual, while in more democratic ecosystems it is distributed (De Noni, Ganzaroli, and Orsi 2013). In the latter case, control of the OSS project is usually maintained by a central group of actors who have gained a level of influence by proving merit, building trust, and social capital through contributions to the OSS project. 


\section{Research Design}

This study presents an exploratory multiple-case study (Runeson et al. 2012) that we conducted to investigate four instances of OGD ecosystems, see Table 1. Case 1 (JobTech Dev) was selected due to the first author's prolonged engagement in its platform provider. Cases 2-3 (Trafiklab and HSL DevCom) were selected due to their characteristics as what we consider as mature examples of OGD ecosystems (based on our provided definition), but also due to their Nordic context where the government has a central role and high level of trust. Case 4 (The City of Chicago) was selected purposely to get an ecosystem with a general domain (municipality) compared to the other examples of labour market and public transport, and also to provide an example beyond the Nordic context.

The actors in the four ecosystems involve organizations and individuals from both public and private sectors, civic society, academia, and private citizens. The unit of analysis is the ecosystems' collaboration around OGD published on the ecosystems' underpinning platforms along with any related OSS and standards.

To be able to analyze the collaboration, we used a model from our earlier work (Linåker and Runeson 2020a) to visualize roles and structure of the studied ecosystems' governance. The model is commonly used for describing the governance in OSS ecosystems (Nakakoji et al. 2002) and was recently applied to a government-initiated OSS ecosystem (Robles, Gamalielsson, and Lundell 2019).

The research effort was initiated with Case 1, where the first author of this study is embedded as an action researcher, as a part of a long-term research project. The researcher was hence able to generate in-depth knowledge through prolonged engagement along with access to extensive documentation. The documentation, field notes and three semi-structured interviews are used to triangulate findings (for questionnaire, see supplementary information ${ }^{3}$ ). To ensure construct validity (Runeson et al. 2012), we based the questionnaire on earlier work about assessing the governance structure of software ecosystems (Alves, Oliveira, and Jansen 2018; Jansen 2020). The interviewees were the platform's product owner, community manager, and policy strategist.

Table 1: Characteristics of the four OGD ecosystems studied.

\begin{tabular}{|l|l|l|l|l|l|}
\hline Case & Ecosystem & $\begin{array}{l}\text { Platform pro- } \\
\text { vider }\end{array}$ & Scope & Founded & Owners \\
\hline 1 & JobTech Dev & $\begin{array}{l}\text { Swedish Public } \\
\text { Employment } \\
\text { Service }\end{array}$ & $\begin{array}{l}\text { Swedish labor } \\
\text { market }\end{array}$ & 2018 & $\begin{array}{l}\text { Swedish national } \\
\text { government }\end{array}$ \\
\hline 2 & Trafiklab & Samtrafiken & $\begin{array}{l}\text { Swedish pub- } \\
\text { lic transport }\end{array}$ & 2011 & $\begin{array}{l}\text { Regional public } \\
\text { transport author- } \\
\text { ities and pri- } \\
\text { vately owned } \\
\text { transport opera- } \\
\text { tors }\end{array}$ \\
\hline
\end{tabular}

https://github.com/johanlinaker/supplementary-material-jedem-2020 


\begin{tabular}{|l|l|l|l|l|l|}
\hline 3 & HSL Devcom & $\begin{array}{l}\text { Helsinki Re- } \\
\text { gional Transport } \\
\text { Authority (HSL) }\end{array}$ & $\begin{array}{l}\text { Helsinki re- } \\
\text { gional public } \\
\text { transport }\end{array}$ & $\begin{array}{l}\text { Municipalities in } \\
\text { the region }\end{array}$ \\
\hline 4 & City of Chicago & City of Chicago & $\begin{array}{l}\text { City of Chi- } \\
\text { cago }\end{array}$ & City of Chicago \\
\hline
\end{tabular}

Data was gathered from Case 1 before any intervention was introduced from the action research. To avoid researcher bias, peer-debriefing between the first and second authors was performed continuously (Runeson et al. 2012).

For Cases 2-4, data were also gathered through semi-structured interviews (see Table 2), using the same questionnaire. All interviews were audio-recorded with additional notes taken. A threat regarding the reliability concerns that only the first author conducted the interviews (Runeson et al. 2012). To mitigate the threat, member-checking was performed in both cases, where synthesized findings were presented to all interviewees who were asked for correctness, misinterpretations, and redundancy.

A series of apriori codes were defined based on our definition of an OGD ecosystem as presented in Section 1.

- Scope and purpose of ecosystem

- OSS projects shared as part of platform

- Open Data shared as part of platform

- Stakeholder and ecosystem actors

- Ecosystem governance

- Collaborative aspects related to OSS

- Collaborative aspects related to Open Data

- Collaborative aspects related to standards

Table 2. Interviewees from the four cases JobTech Dev, Trafiklab, HSL DevCom and City of Chicago.

\begin{tabular}{|l|l|l|l|l|}
\hline ID & Role & Ecosystem & Employer & $\begin{array}{l}\text { Years with } \\
\text { ecosystem }\end{array}$ \\
\hline 1 & Product owner & JobTech Dev & SPES & 2017- \\
\hline 2 & $\begin{array}{l}\text { Community } \\
\text { manager }\end{array}$ & JobTech Dev & SPES & $2018-$ \\
\hline 3 & Policy strategist & JobTech Dev & SPES & $2018-$ \\
\hline
\end{tabular}




\begin{tabular}{|l|l|l|l|l|}
4 & Product manager & Trafiklab & Samtrafiken & 2019- \\
\hline 5 & Data specialist & HSL DevCom & HSL & $2010-$ \\
\hline 6 & Unit manager & City of Chicago & City of Chicago & $2013-2018$ \\
\hline
\end{tabular}

Synthesized findings are presented per case in Section 4 where codes have been abstracted into the following four categories:

- $\quad$ General Background (C1)

- $\quad$ Platform Content (C2-3)

- $\quad$ Ecosystem Governance Structure (C4-C5)

- $\quad$ Orchestration and Collaboration (C6-C8)

Quotes from the interviewees (see table 2) are used to provide further context and nuance to the findings. The cases were then cross-analyzed based on the apriori codes which resulted in a conceptual model of OGD ecosystems. The model consists of eight aspects, each with a number of attributes, which emerged from the analysis. The model is visualized is Fig. 2 and presented in detail in Section 5.

\section{Results}

This section summarizes the results from the four studied cases, JobTech Dev, Trafiklab, HSL DevCom, and the City of Chicago. 
Figure 1: Overview of the governance structure of the four cases in this study, JobTech Dev (upper left), Trafiklab (upper right), HSL DevCom (lower left), and City of Chicago (lower right), based on the governance model presented in earlier work (Linåker and Runeson 2020a) (in turn adopted from Nakakoji et al. (Nakakoji et al. 2002)). The core is occupied by the platform provider who controls development and data shared on platform. Ecosystem actors are divided among a number of layers specific for each ecosystem. Actors important to the platform provider and ecosystem, and with high influence on the platform are close to the core. Influence and activity decrease for each additional layer. The closer to the core, the more influence they have on the platform

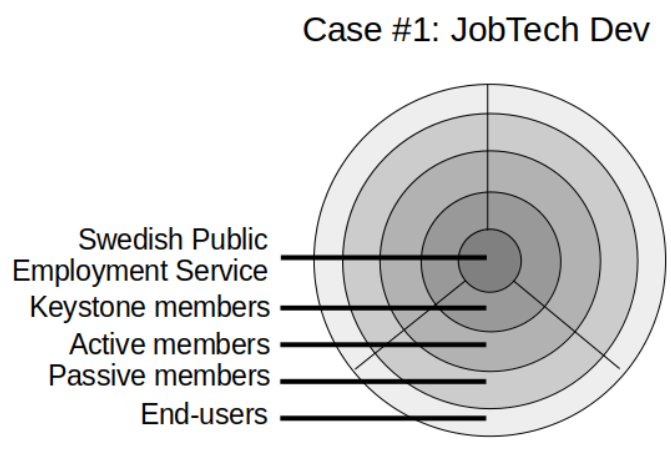

Case \#3: HSL DevCom

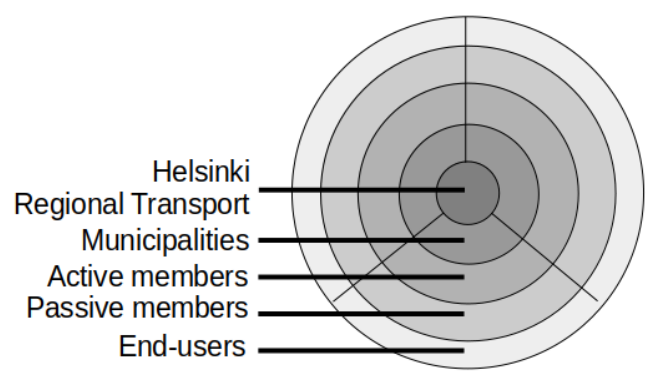

Case \#2: Trafiklab
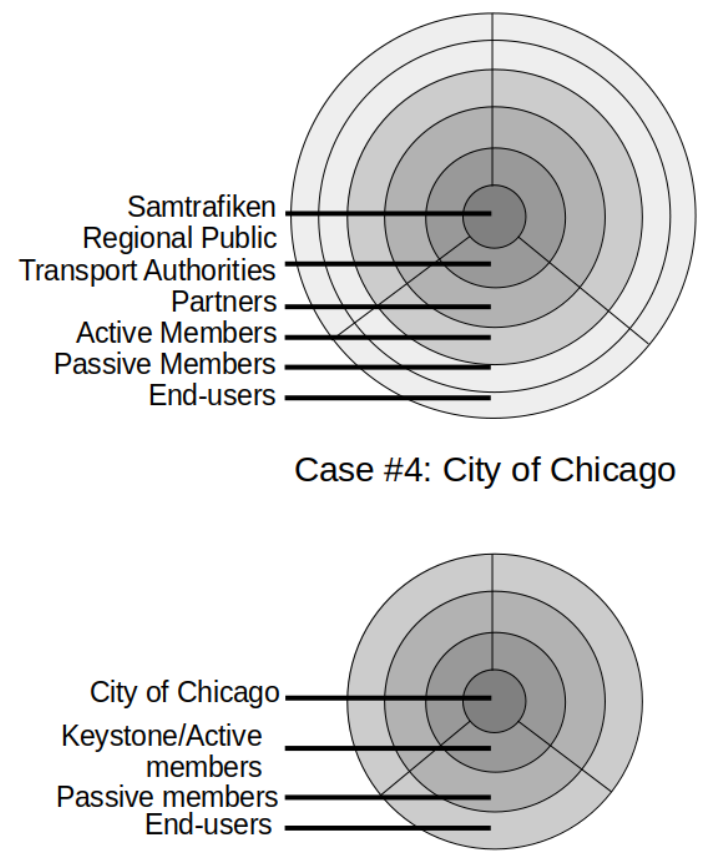

\subsection{Case 1: JobTech Dev}

\subsubsection{General Background}

JobTech Dev is an ecosystem, initiated in 2018, bringing actors together which operate within or in relation to the Swedish labor market, to collaborate on a common platform of OGD, connected APIs, and complementary OSS projects. The ecosystem and its platform are developed and orchestrated by the Swedish Public Employment Service (SPES), a Swedish national government agency responsible for enabling the match making between job-seekers and employers on the labor market. JobTech Dev was created as a part of this mission to enable the actors in the ecosystem to accelerate their innovation process, improve their services, and thereby improving the digital match making on the labor market.

\subsubsection{Platform Content}

The platform consists of three parts: Jobs, Taxonomy, and Career. 
Jobs is a service intended to collect job advertisements on the Swedish labor market through a collaboration with several ad providers and make the ads available through a common API.

Taxonomy is a collection of skills and job titles and relationships between them. The data set is developed and maintained within SPES. By opening up the data through APIs, the actors in the ecosystem are enabled to "speak the same language" enabling, for example, improved reporting and statistics and match-making between job advertisements and job-seekers' resumes. The data is released under the CC0 1.0 license ${ }^{4}$.

Career is (unlike Taxonomy and Jobs) not OGD. It is a service where job seekers can store their resumes on a central location in an encrypted format. The job seekers can grant and withdraw permission to organizations, for example, recruitment firms, social networks, and insurance firms to access their information. The service is based on the MyData principles ${ }^{5}$ and enables job seekers to only have to maintain one copy of their resume and to distribute and manage their data with kept control over their integrity and privacy.

The source code for the APIs is available as OSS as are the algorithms. To lower adoption barriers to the data, example applications are also developed and released as OSS. Members consuming the data, specifically startups, have acknowledged the value provided by these examples as it helps them understand use-cases and accelerate their development.

\subsubsection{Ecosystem Governance Structure}

In terms of operations, the members of the ecosystem can generally be categorized within one of the areas: 1) recruiting and staffing firms, 2) education and guidance providers, 3) national, regional or local governments, 4) workers' unions, 5) employers' associations, 6) job advertisers, and 7) job seekers. Depending on the category, a member's interest in the platform may be limited to certain parts of the platform.

To visualize the governance structure, we use a governance model presented in earlier work (Linåker and Runeson 2020a) (in turn adopted from Nakakoji et al. (Nakakoji et al. 2002)). SPES is positioned in the center as the platform provider orchestrating and governing the ecosystem (see Fig. 1, upper left). SPES ultimately decides on requirements and a road map for the platform, including what data to make available, when, and how. They perform the necessary development and maintenance and provide the infrastructure needed to access and use the data.

In the layer closest to SPES are the members whose opinions may be considered as extra important for SPES in terms of developing the platform and growing the ecosystem. These members may, for example, have a large user base, or valuable competencies and resources, and thereby contribute to the health of the ecosystem. In the second layer are the general members and in layer three the end-users. Each layer is viewed to potentially consist of members from all types of operations.

\footnotetext{
https://creativecommons.org/publicdomain/zero/1.0/

https://mydata.org/guiding-principles/
} 


\subsubsection{Orchestration and Collaboration}

Due to the limited internal resources, SPES cannot maintain formal and direct relationships with all ecosystem members. Teams carrying out development inside SPES therefore primarily work and communicate through close relationships with the key members to optimize the impact. However, SPES is striving to adopt an open development model and maintain an open dialogue where the whole ecosystem (including all layers in Fig 1) can influence the direction of the platform.

Anyone can, for example, request and discuss a new feature, an API, or data set through an asynchronous open communication platform or by attending occasional meetups arranged by SPES. It is also possible to contribute to the development, although external contributions so far have been limited to bug reports and feature requests. The intention however is to encourage and enable members to contribute to both new and existing projects. Non-code contributions are however more common, for example, in the form of validating the quality and performance of the OSS. One example constitutes algorithms used to remove duplicate job ads as well as assign statistical identifiers to the job; ads that are collected and then made available through an API. External actors, including the Swedish statistics agency, have thus been able to test and validate the quality of the algorithms and whether they can trust the results the algorithms produce.

In terms of data, there are examples - although limited - of members producing and contributing directly to the platform. One example is a set of soft skills and their relationship to different job titles, which was contributed to the Taxonomy part of the platform. Processes are not yet established for how these types of contributions should be managed. In this case, a formal contract was established between the two parties.

Close collaborations and direct dialogues with key members have been important to establish the ecosystem and gain general acceptance. For example, the collection of all job-ads on the market and making these freely available, made incumbents offering recruitment and staffing services initially question the intent from SPES as well as the suggested benefits. SPES views the commoditization of job-advertisement data, as with the ecosystem at large, as a way to push the actors forward who are working with digital match making and guidance services, nurturing innovation and lowering barriers to entry, for new actors.

After a more than two-year process, even the more conservative incumbents started to accept the ecosystem and see potential benefits with it. A formal collaboration was initiated between SPES and the ten largest job advertisement providers, where they agreed to allow job-advertisements to be collected. Once collected, the ads are converted to an industry-specific open standard, and then enriched with metadata such as the date of publishing and a deadline for applications. A compromise was reached to only provide a "stub" of the advertisements, meaning that only the job title, metadata, and a link to the original advertisement would be provided through the platform's API.

SPES works actively to adopt or develop open standards when needed to promote and improve interoperability between the actors in JobTech Dev as well as towards the outside. One example is the adoption of the HRO open standard ${ }^{6}$ that was adopted for the way individuals' resume data is

https://www.hropenstandards.org/ 
distributed. Also, to make their MyData-based implementation underpin the Career-initiative, they have initiated a collaboration for defining interfaces and API:s within the MyData community ${ }^{7}$

\subsection{Case 2: Trafiklab}

\subsubsection{General Background}

Trafiklab is an ecosystem, initiated in 2011, that brings actors within the Swedish public transport sector together to collaborate on a platform with open traffic data, connected APIs, and complementary OSS projects. The ecosystem's vision is to facilitate the creation of new services that makes it easier and more attractive to travel with public transport. The ecosystem and its platform are developed and orchestrated by Samtrafiken, a corporate entity co-owned by all the regional public transport authorities and most of the commercial transport operators in Sweden. Operators also have the option of being partners to Samtrafiken without being co-owners.

\subsubsection{Platform Content}

The platform consists of data sets and APIs, either maintained by Samtrafiken or independently by members of the ecosystem. All data hosted on the Trafiklab-platform is released with a custom license based on the principles of the CC0 1.0 license.

Four APIs provide static and real-time data on public transport, related to, for example, timetables and interruptions. This data is currently made available in two types of standard formats, maintained by Samtrafiken and gathered from the regional public transport authorities and private operators, following a government directive. Two further APIs provide timetable data for a trip-planner, an externally procured product that "... is offered for free as-a-Service" (I4) to the ecosystem.

Certain APIs are maintained by other organizations, both public and private, and made available on the Trafiklab platform. Data includes timetable and service data from Stockholm Public Transport and traffic information from the Swedish Transport Administration. The platform also links to related APIs that are maintained and hosted by other organizations. These include data from regional public transport authorities, local counties, and private entities.

In regards to OSS, they currently have a number of software development kits and example applications available. They intend to develop a new OSS trip planner and share their APIs as OSS along with the different parts of the platform.

\subsubsection{Ecosystem Governance Structure}

In terms of operations, the members of the ecosystem can generally be categorized within one of the areas: 1) regional public transport authorities, 2) private and publicly owned train operators, 3) national, regional, and local governments, 4) private bus operators, and 5) private product and service providers. Plans include integration with related actors, such as taxi operators and rental-service providers of, for example, cars and bikes.

7

https:/ / mydata-infrastructure-project.github.io/ 
In terms of the governance structure, Samtrafiken is positioned in the center as the platform provider orchestrating and governing the ecosystem (see Fig. 1, upper right). Outside of Samtrafiken in the first layer are regional public transport authorities. As these are formal owners of the platform, they have a strong influence on the direction of Trafiklab. In the second layer are the formal partners to Samtrafiken which may include actors with different types of operation. The third layer primarily consists of private product and service providers, while end-users are positioned in the fourth layer.

\subsubsection{Orchestration and Collaboration}

Close relationships are maintained with regional public transport authorities and partners as these are the primary data producers but also consumers. The ecosystem at large can report bugs, ask for help, and request and discuss new features, APIs, or data sets through an asynchronous, open communication platform. Physical meetings can also serve a similar purpose since Samtrafiken frequently hosts hackathons and meetups related to Trafiklab.

As with SPES, Samtrafiken is transitioning to a more open and collaborative way of engaging with its ecosystem, a need identified in earlier research (Rudmark and Hjalmarsson-Jordanius 2019). What they have released thus far has mostly "...been sporadic - we need to have this for that meetup" (I4). I4 adds however, "After this change we will have a clearer strategy regarding open source, where everything we do will be available as open source". One part in this are the "...plans to build an open source journey planner based on the same data as the proprietary version" (I4). Another part in its adoption of more open and collaborative practices, is Samtrafiken move towards opening up its roadmaps, while also discussing a more formal approach where users can request and vote on what data sets should be prioritized. External contributions have thus far been limited to bug reports and feature requests.

Regarding the data, all of the provided data sets originate from data producers within the ecosystem. Depending on the case, Samtrafiken may transform the data to certain standard formats, develop and maintain the necessary APIs, and provide the necessary infrastructure for data consumers. A challenge with growing the ecosystem and gaining new data producers is related to standard formats of the data. For smaller actors, it is an expensive process to transform the data, and for Samtrafiken a recognized risk is that data may be destroyed when transformed between standards. Samtrafiken is, therefore "...developing an input-portal to enable actors that do not have the resources or can afford it, to share their data on Trafiklab and to automate the transformation process" (I4). The portal is specifically intended for actors in areas related to public transport, such as taxi operators and rental-service providers. The input-portal is a result of a long-term investigation conducted by Samtrafiken and its partners into the future potential and needs for public transport-related OGD. The investigation also rendered in a plan to introduce 12 new data sets by 2021.

Other than helping data producers to transform their data into different standards (for example, the GTFS format, an industry standard for public transportation schedules and associated geographical information ${ }^{8}$ ), Samtrafiken and its partners within the Trafiklab ecosystem also collaborate on the development of new standards when needed. A standard for tickets and payment transactions

https://en.wikipedia.org/wiki/General_Transit_Feed_Specification 
was developed "...with the goal of enabling sales of tickets across regional borders which was difficult to solve in existing proprietary solutions" (I4).

\subsection{Case 3: HSL Developer Community}

\subsubsection{General Background}

HSL Developer Community (HSL DevCom) as an ecosystem was created in 2009 as the first data sets were published. However, "...the ecosystem was far from established, for example, we did nothing related to open source software at this time" (I5). The ecosystem brings together actors with an interest in OGD and OSS related to the Helsinki region's public transport (compared to the national focus in the case of Trafiklab). The ecosystem and its platform are developed and orchestrated by the Helsinki Regional Transport (HSL as per the Finnish translation) authority, a local joint authority coowned by nine municipalities in the Helsinki region of Finland. HSL is, among other things, responsible for the planning and organizing of the region's public transport system.

\subsubsection{Platform Content}

The platform consists of several data sets and connected APIs as well as a number of related OSS projects, central to the ecosystem actors, both in terms of internal operations and as customer-facing applications. All data is made available under the CC-BY 4.0 license $^{9}$.

The main part of the platform is related to the Journey Planner ${ }^{10}$, an OSS application for travelers planning their journeys within the region's public transport system. The Journey Planner was HSL's first OSS project, piloted in 2013, where the core consists of the Open Trip Planner OSS project. "The user interface we have built ourselves, we use Open Trip Planner for the route planning and any outwardfacing API:s" (I5). The Journey Planner is developed in collaboration with the Finnish Transport Agency and Waltti, a public transport travel card collaboration in Finland. "HSL leads the development while the other two partners mainly contribute financially" (I5). The platform further provides four underlying open APIs which provide $i$ ) itinerary- and timetable data, $i$ ) geocoding data with addresses and coordinates, iii) route suggestions between two coordinates, and vi) the underlying map data. The data for $i-i i i$ is produced by HSL while the map data for $v i$ is generated from OpenStreetMap (OSM), after a number of operations has been performed such as "filtering of the data, creation of grid patterns, and visualizations using [HSL's] own map style" (I5).

A second main OSS application, also using the same underlying APIs, is the Map Generator11, a web-based tool for generating, for example, route maps, traffic bulletins, and stop posters. The development of the Map Generator is mainly performed by HSL.

Other APIs that are provided via the platform cover data related to: 
- Bluetooth beacons installed on buses, bus stops, and terminals for trains and subways: One use case is to maintain an overview of the number of passengers waiting for or currently undergoing a ride. Another use case is to provide "...better information regarding the current ride they are taking, and provide traffic information and potential alternative traveling options" (I5). The data is produced and published by HSL.

- $\quad$ Trips made with city bikes in Helsinki: The data includes information related to the trips start and endpoints and is mainly used for analysis. The data is produced by City Bike Finland and contributed to HSL once a month who publish it on the platform.

- $\quad$ Car park information of commuters: The data is produced by municipalities and operators via a browser-based user interface on HSL's website. The data is then published via the API on the platform by HSL.

\subsubsection{Ecosystem Governance Structure}

Similar to Trafiklab, members of the ecosystem can in terms of operations generally be categorized as: 1) national, regional, and local transport authorities 2) governments, but also 3) private bus 4) train operators, and 5) private product and service providers. As the use of the Journey Planner stretches beyond Finland, members of the ecosystem also cover Norwegian, Swedish, German, and Italian markets (to various extents).

In terms of the governance structure, HSL is positioned in the center as the platform provider (see Fig. 1, lower left). Outside in the first layer are the nine municipalities that co-own HSL and thereby have the formal ownership of the platform and a strong influence on the direction of its development where "...the larger municipalities have a bit more influence" (I5). The formal partners to HSL are in the second layer, in relation to the Journey Planner, i.e., the Finnish Transport Agency and Waltti, which help to fund the development and maintenance of the Journey Planner and its underlying APIs. The active members of the ecosystem are in the third layer, which primarily include the national and international users of the Journey Planner, as well as large consumers of the data APIs such as Google who incorporate it in their search and map products. The fourth layer contains the more passive, private product and service providers, while end-users are positioned in the fifth layer.

\subsubsection{Orchestration and Collaboration}

HSL has, since the launch of the Journey Planner, had an open-by-default perception, that most of what they develop should be available as OSS. "We had this proprietary solution we needed to replace, and we had thoughts on it, that we wanted to try it as an open source and use OpenStreetMap, then we found the Open Trip Planner project which does a lot of the things we need" (I5). Hence, the authority has a large set of OSS projects available on their GitHub account. Among these, it is only the Journey Planner that benefits from external contributions. Due to deviations in interest and agendas, there is a branch of the Journey Planner that is being developed and maintained by the Norwegian Transport Authority. Both sides are however working towards integrating the two versions of the Journey Planner.

I5 reflects that the HSL has generally been focused on the development and hoped that the external contributions would come automatically with time, which, according to the interviewee only 
holds for the Journey Planner OSS project. However, in an upcoming large development project related to their master data registry, they have an "...ambition to establish a collaboration with other public transport operators or organizations from start" (I5).

In terms of the OSS projects, there are issue trackers for each specific project on GitHub. Bug reports or feature requests are however limited, with the Journey Planner as an exception. However, there is no general open issue tracker for the platform and its APIs that is actively used. Instead, the e-mail is the most common option along with HSL's social media accounts at Facebook and Twitter, which can be considered as the main communication channels used towards the ecosystem at large. They do use Slack, a synchronous communication platform which, however, is closed and exclusive for the main partners and international users of the Journey Planner OSS project. Physical meetings are frequently used to communicate with the ecosystem, present plans, and collect requirements, typically by speaking at conferences or partnering with external hackathons.

In terms of data, there is a mix of data produced by HSL (for example, itinerary- and timetable data, and Bluetooth beacon data), by actors within the ecosystem (for example, geocode data from national authorities and car park data from the municipalities), and by ecosystems external to HSL DevCom (for example, map data from OpenStreetMap). Some data is automatically collected such as geocode data from municipalities or map data from OpenStreetMap, whilst other data may be shared by the ecosystem actor through technical infrastructure (car park data) or directly via mail (city bike trips data).

To share maintenance and reduce the amount of internal edits needed, HSL has a full-time editor and some part-time resources available as needed that contribute to OpenStreetMap. Edits may, for example, include the move or addition of a bus stop, or the correction of street names. There have been contributions of larger data sets to OSM as well. These are however limited due to that HSL considers the current contribution process expensive in terms of resources, and due to conflicting licenses. "We have to provide the national road database with bus stop data licensed under CC-BY-4.0 and can therefore not base all of our data on OSM, because that would "infect" the data we export with OSM's $[O D b L]$ license".

As with Samtrafiken, HSL promotes the use of the GTFS standard "... after a request from Google, however, it was rather inevitable, as it has become a de facto format, even though there are other standards used as well" (I5). No examples were given regarding development of new standards or specifications within the ecosystem.

\subsection{Case 4: City of Chicago OGD ecosystem}

\subsubsection{General Background}

The City of Chicago's OGD ecosystem was initiated in 2011 through the launch of the city's open data program and data portal. The ecosystem at large covers the whole city, including its citizens, NGOs, and companies. The main goal is to make the city's internal data useful to and consumable by the public. The first driver of the initiative was to increase transparency, which with time evolved to a higher focus on enabling social and economic development. Another important motive is to 
engage the public to improve their community. Citizens with a tech background see it as a way to "pay back to [the] community and be able to volunteer some of their time by using their skills" (I6).

\subsubsection{Platform Content}

The platform consists of about 1400 different data sets available through APIs. The data springs out of the administrative processes that are carried out by the City of Chicago. There is hence a wide variety in the type of data spanning from every permit issued by the city, every crime reported, to every trip taken via mobility and ride-sharing operators. The most popular data set however was the "the list of salaries and all the employees who worked for the city" (I6). The data is released under a custom licence ${ }^{12}$.

In terms of OSS, the city has about 80 published OSS projects on their GitHub account, where a smaller number is considered more active and used than others. A common theme is that there is a connection to the data published on the city's data portal. Two of the more popular projects are made up of algorithms, that together with data from the portal, support decisions on where to send food inspectors in the city, and predicting E. coli levels in Lake Michigan, respectively. Another project regards OpenGrid ${ }^{13}$, a web-based map that allows the user to explore multiple data sets simultaneously.

\subsubsection{Ecosystem Governance Structure}

The members of the ecosystem surrounding the OGD and OSS provided via the city's platform are cross-sectoral, involving research institutions, companies, Non-Governmental Organizations (NGOs), journalists, and citizens. As there is a wide variety in the data sets provided, there is no specific industry or area to classify these members in (as with the other cases presented in this paper, such as actors) connection to the Swedish labor market, as in the case of JobTech Dev.

Considering the governance structure, the city is in the center position as the platform provider (see Fig. 1, lower right). At the closest layers, they don't prioritize certain groups before others (as with SPES for example) but listen to those actors that are actively communicating and collaborating with them. A representation can be found among all sectors listed above. Journalists, for example, are highlighted as one category who often interact and request the release of data sets related to their investigations. NGOs are another important type of actor as they often interface and reach larger groups of citizens, either in general or around certain topics. Research institutions are also of importance as they are often be a part of developing OSS applications and data science projects using the published data. Certain companies, including startups and incumbents, may be more vocal than others, depending on their dependence of the data. Some citizens may also be considered as keystones by frequently filing public record requests.

As with the other cases, the more passive users of the data are in the proceeding layers, followed by the end-users of the many applications that are created by the actors in the middle layers.

12 https://www.chicago.gov/city/en/narr/foia/data_disclaimer.html

13 https://github.com/Chicago/opengrid 


\subsubsection{Orchestration and Collaboration}

As with HSL, the City of Chicago has initiated a number of OSS projects that are available on the city's GitHub account. Of these, only parts are developed and maintained actively and are getting external contributions. In this open collaboration, research institutions make up important partners as they, in turn are incentivized to collaborate with the government and also share the goals of the city in terms of "creating broad public value" (I6) through the development of OSS. External grants have been a common tool used to fund this collaboration and thereby the development of OSS projects such as OpenGrid.

Also, companies constitute important partners, as highlighted in the OpenGrid project. Their contributions are in part development procured by the city, but also come as an effect, as companies can offer and sell support for the OSS project to other customers (which was actively encouraged by the City of Chicago). Another successful project in terms of external contributions is the algorithm for measuring E. coli levels in Lake Michigan. The project, which was led by the city with a staff of eight people, received "over a thousand hours of in-kind contributions" (I6).

For OSS projects that are actively maintained, the city tries to publish both roadmaps and documentation, and use the collaborative infrastructure offered by GitHub. I6 describes how they with time have "figured out what sticks, and then where you actually have to pay your attention to" and also "take lessons from others and see how other [OSS] projects organize themselves. For prioritized projects such as OpenGrid, the city organizes public meetings, physically and virtually, where the city can solicit feedback and present the roadmap so that "people can contribute to it and in a way that makes sense" (I6). In certain cases, working committees would be set up to facilitate collaboration around specific topics. A common theme among the OSS projects is that they in some way make use of the data and enable the analysis and exploration of it. The city views OSS as a mechanism for interacting with the citizens around the open data program and enables them to make use of the data, and also contribute back.

The data shared to a large extent, originate from within the city. However, it is not the city's intention ...to accumulate all data related to the City of Chicago (I6). Instead, they try "...to be pragmatic, add things of value [and] be able to meet people's needs" (I6). Hence, there are in general no specific domains that are considered in terms of sharing data. Sometimes there are exceptions when a specific initiative is highlighted, like clean energy and sustainability, where some specific data sets would be released (I6). Not all data originates from within the city, however. Some data sets are gathered from the national government, such as census data, population data, and socioeconomic data, and then broken down into the city's community areas. Mobility data generated from services such as taxi, ride sharing, and bike rental, is also collected and published due to legal requirements.

The city has attempted to collaborate on certain data sets with the public by publishing the data sets on GitHub, similar to what is done with the source code in OSS projects. These data sets are mainly connected to geographic entities such as "bike paths, streets, bike racks, [as] these are things very much in the public where [the citizens] actually might be able to see something and know it before the government knows about it" (I6). One example of external contributions regarded footprints of the city's buildings. This data set was in turn contributed to by citizens via the OpenStreetMap. Although a successful example, the city did "not experience GitHub as a good platform for sharing geospatial data and 
contributing data" (I6). Alsoas the interest from the public was limited, they did not pursue this model of collaboration any further, beyond the initial initiatives.

The city uses open standards when possible to enable interoperability between similar data sets from other cities, for example, through the use of GTFS related to public transport (similar to Samtrafiken and HSL) and the Mobility Data Specification related to mobility services. They also engage and codevelop new standards for how to publish data when needed. One example referred to a collaboration between a number of cities on how to commonly display the location and availability of flu shots.

\section{Analysis and Discussion}

JobTech Dev (Case 1), Trafiklab (Case 2), HSL DevCom (Case 3), and the City of Chicago (Case 4) present both similar and differentiating attributes as OGD ecosystems. In this section, we break these attributes down into several aspects, together forming a conceptual model emerging from the analysis (see Fig. 2) along with recommendations for platform providers to consider when (re)designing their OGD ecosystems, to foster collaboration and the sharing of resources. Each aspect of the model is explained and contrasted individually below, highlighting its different attributes that were identified in the four cases. 
Figure 2: A conceptual model for OGD ecosystems consisting of eight main aspects and connected attributes as explained in section 5. Each attribute has the corresponding case number within brackets where it was identified.

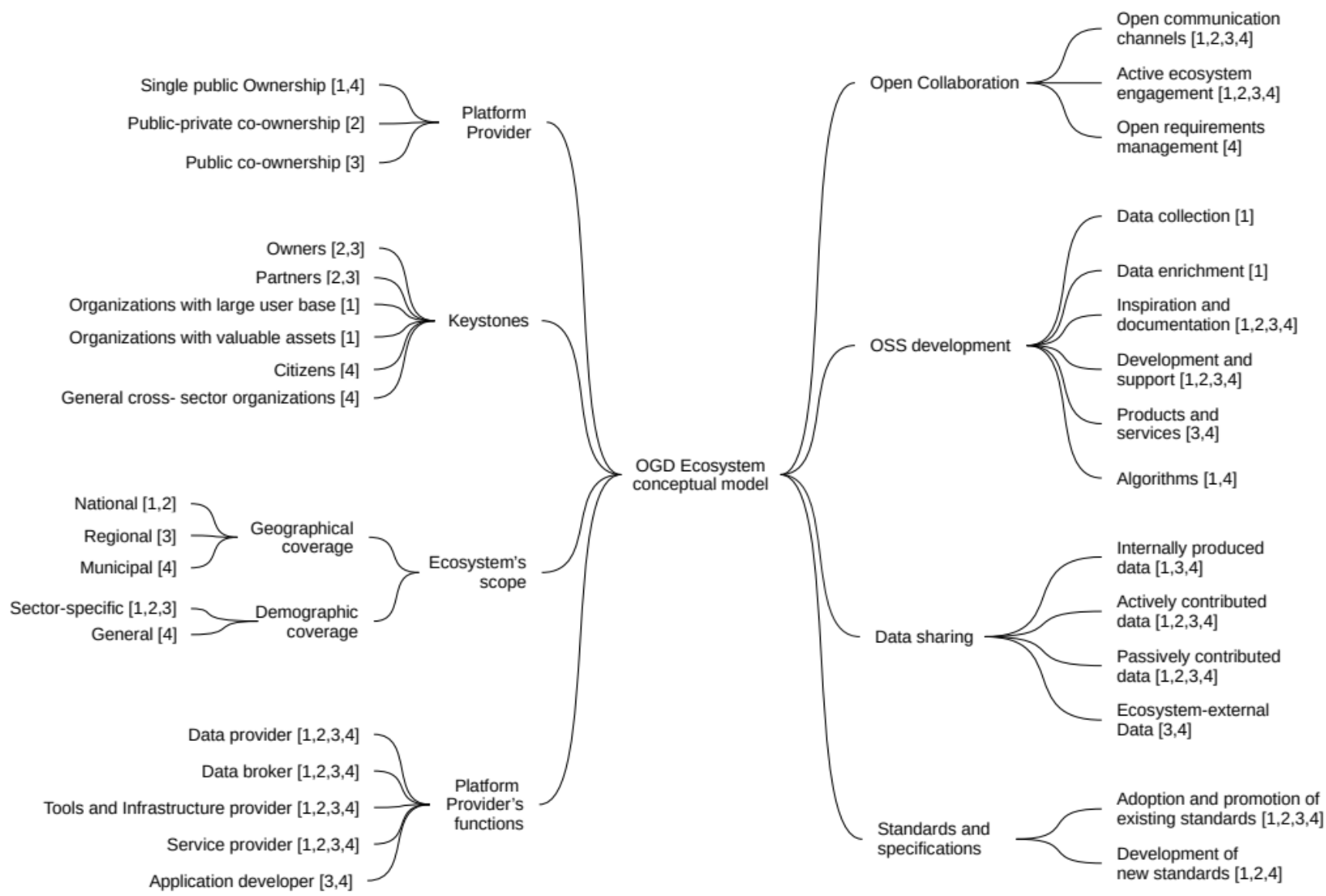

\subsection{Platform Provider and Keystones}

Among the studied cases, we observed three different types of platform providers based on the owner(s) it represents; Single-public ownership, Public-private co-ownership, and Public co-ownership. The single-public ownership is represented by the case of JobTech Dev and the City of Chicago, which are governed by a single public entity, namely SPES and the city government, respectively. They decide who the keystones and main stakeholders are, and how they may influence the content and development of the platform (cf. keystone-based governance (Dal Bianco et al. 2014)). In SPES' case, they prioritized those whom they consider to be large and important actors, while the City of Chicago maintains a broader definition of whom they consider as keystones. This freedom to choose allows them to potentially move quickly, but also has the risk that their direction can easily change due to change in the national and local government, respectively. This risk could be a concern for both existing, and new members in whether they can trust the direction and stability of the platform and ecosystem, and thereby if they should invest in platform integrations. 
The public-private co-ownership model is represented by the case of Trafiklab, which is governed by Samtrafiken, an organization co-owned by all the Swedish public transport authorities along with a number of privately owned public transport actors. The co-ownership of the platform provider provides a somewhat neutral body among the public and private actors, who some may consider to be competitors. This consortium-based governance structure (Dal Bianco et al. 2014) may be a way to ensure the critical aspect of trust in the platform provider's commitment and a guarantee for the long-term stability and collaboration within the ecosystem (Susha et al. 2020). Similarities may be drawn from the role of data trusts (Coyle, Diepeveen, and Wdowin 2020) as well as OSS foundations (De Noni, Ganzaroli, and Orsi 2013) as proxy-organizations and neutral homes for data and OSS projects respectively. In these organizations, actors can collaborate and invest together in a way that benefits them all, and with clear charters stating how the project will be technically governed. A risk with this form of ownership, however, is that the platform provider may not be able to receive government funding as it would benefit the private actors who are part of its the ownership (in contrast to those who are not).

The public co-ownership model is represented by the case of HSL DevCom, which is governed by the HSL, an organization co-owned by nine out of fourteen municipalities in the Helsinki Region. The remaining five have the option to join the collaboration should they prefer. This model allows for the municipalities to collaborate, as with Trafiklab, through a consortium-based governance structure (Dal Bianco et al. 2014) and also provide joint services and operations that are specific to their assignments as public entities.

Recommendations: Based on the studied cases, we advise public entities acting as platform providers in an OGD ecosystem, to:

- $\quad$ consider how to best enable collaboration and engagement from keystone players in the ecosystem through platform ownership: If the role of a platform provider is concentrated to a single public entity (single-public ownership), it needs to enable dialogue and influence from keystones, for example, through an open collaboration. If ownership is distributed among the keystones, for example, a number of public entities (public co-ownership), or a number of public and private entities in collaboration (public-private co-ownership), collaboration may be ensured through formal organizational structures.

- $\quad$ identify and build trustful relationships with the keystones of the ecosystem as their engagement and success is important for the overall health and success of the ecosystem: Actors that constitute as keystones will vary, but may include owners, partners, organizations with large user bases or other types of valuable assets, citizens, or general cross-sector organizations.

\subsection{Scope of Ecosystem}

The ecosystem can cover different scopes, both in terms of geographical and demographic coverage. The scope is in part defined by the platform provider who creates, governs, and facilitates the ecosystem. They set the vision and focus for the platform and highlight use cases it should address (Iansiti and Levien 2004). Returning to our definition of an OGD ecosystem (see Section 1), we see how this 
connects to the common interest on which actors base their relationships. Continuing from this perspective, the scope is also partly defined by the actors who join; since without them there is no ecosystem. The vision and scope of the ecosystem is therefore something that potentially evolves organically with time.

Looking at geographical coverage, we can notice that JobTech Dev and Trafiklab have national coverage of Sweden, while HSL DevCom and the City of Chicago have a more regional and municipal coverage on the municipal government. However, even though the main actors may be limited geographically, the same limitations do not have to concern all actors within the ecosystem. In the case of HSL DevCom, Google and Apple, for example, are interested in the public transport on a global level, not just in the Helsinki region in Finland.

Considering the demographic coverage, Trafiklab, HSL DevCom, and JobTech Dev have a sectorspecific focus on public transport and the Swedish labor market, respectively. However, even though the data may be limited, use cases don't have to be. These can be generic or positioned in a separate domain where the data from the platform in concern, is only one piece of the puzzle. In contrast, the City of Chicago has a very general focus in terms of its ecosystem as the OGD and OSS they provide may relate to any part of the city's many services and functions.

Recommendation: Based on the studied cases, we advise public entities acting as platform providers in an OGD ecosystem to:

- maintain a clear vision for the ecosystem, but at the same time allow for the ecosystem's actors to influence and buy in on the vision. It is important to also consider actors whose use case may stretch beyond the intended geographical and demographic coverage of the ecosystem.

\subsection{Platform Provider's Functions}

Looking at the functions, or responsibilities taken on by the platform providers in the investigated ecosystems, they cover many of the roles as reported in the literature (Immonen, Palviainen, and Ovaska 2014; Lindman, Kinnari, and Rossi 2015). Besides being data providers, all four platform providers may be described as data brokers as they gather, promote, and distribute data from third-party (Immonen, Palviainen, and Ovaska 2014) (cf. data intermediaries (Susha et al. 2020)), but also data transformers as they transform data between different standards based on ecosystem needs (Lindman, Kinnari, and Rossi 2015). An example of the latter can be found in the case of Trafiklab where Samtrafiken transforms incoming data to the GTFS format.

Another important role that all platform providers take on is that of tool and infrastructure providers, as they develop supporting tools, frameworks, and example applications for their ecosystems, as well as infrastructures to enable the collection and distribution of the data (Immonen, Palviainen, and Ovaska 2014).

Furthermore, all four platform providers also take on the role of service providers, as they, each to a different extent, provide services based on the data they publish. As examples, both HSL and Samtrafiken both provide their respective journey planners, the City of Chicago provides OpenGrid as a service to allow users to explore the OGD published on their data portal, and SPES enables 
individuals to store and distribute their resumé-data to recruitment platforms via their Career initiative. In the case of HSL and the City of Chicago, they also take on the role of application developers as they develop and provide the applications (on which they base their services) as OSS.

Hence, to stimulate and enable collaboration in OGD ecosystems, the platform provider is required to perform multiple functions, which otherwise may be divided among multiple actors (Immonen, Palviainen, and Ovaska 2014; Lindman, Kinnari, and Rossi 2015). In contrast, when entering an existing ecosystem, an organization may consider taking a peripheral, less complex role (Rudmark and Hjalmarsson-Jordanius 2019). This is, however, a trade-off between influence on the platform development and value capture, i.e., if the organization's goals can still be achieved.

Recommendation: Based on the studied cases, we advise public entities acting as platform providers in an OGD ecosystem to:

- consider the needs of the ecosystem's actors and take on an active and multi-functional role in order to stimulate and enable collaboration in the ecosystem. Such functions may include providing and brokering data between actors, as well as providing tools and infrastructure for adopting and processing the data, and related services and applications to enable use cases involving the data.

\subsection{Open Collaboration}

To enable an open collaboration within the ecosystem (Linåker and Runeson 2020b), the platform provider itself needs to open up their processes and communication for the ecosystem using appropriate social and technical boundary resources (Dal Bianco et al. 2014; Ghazawneh and Henfridsson 2013).

Open communication channels are one example of such resources, through which the platform provider and ecosystem actors can collaborate and communicate with each other (Feller, Fitzgerald, and others 2002). All four platform providers have such channels, either synchronous or asynchronous, complemented with alternative channels such as email, social media, and support desks. These channels usually serve as places for posting questions, asking for support but also reporting bugs, and requesting new functionality or data.

Open requirement management is another key boundary resource that includes openly communicating what requirements the provider is working on (Ernst and Murphy 2012). It also involves enabling ecosystem actors to comment as well as request features more formally, for example, through an issue tracker rather than the communication channels. None of the ecosystems have a central issue tracker but use those available via GitHub in connection to the OSS projects. In terms of communicating more long-term plans, only the City of Chicago publishes roadmaps related to their OSS projects.

Active ecosystem engagement is the third type of social boundary resource, meaning that the platform providers need to communicate actively with their ecosystem actors, and also engage, initiate and facilitate collaborations within the ecosystem (Linåker and Runeson 2020b). Beyond using the open communication channels, physical and virtual meetings such as hackathons, meetups, and 
conferences are commonly used among all the platform providers. This aspect is especially important before releasing a new data set or OSS project. For example, HSL reported struggles with gaining contributions as they have been too focused on the development and less on external engagement. As a counter example one can consider the work that SPES has put into preparing the job-ad collaboration.

Recommendation: Based on the studied cases, we advise public entities acting as platform providers in an OGD ecosystem to:

- enable an open collaboration within the ecosystem through the use of necessary and available social boundary resources, for example, by providing open communication channels, while also facilitating an open requirements management process and actively engaging the ecosystem in the collaboration.

\subsection{OSS Development}

Among the studied cases, HSL has a stronger focus on OSS development as certain OSS projects have a more central role in the HSL DevCom ecosystem and are aimed for the end-users. In the other ecosystems, the OSS projects developed, mainly serve as boundary resources to the developers in the ecosystems (Dal Bianco et al. 2014). In the cases studied, we have identified six categories of OSS projects that platform providers may want to consider initiating and collaborating on:

- Data collection: In the case of JobTech Dev, SPES is employing OSS-based technology (developed and maintained by a third-party supplier) to collect the job-ads from the different job-ad suppliers.

- Data enrichment: OSS can also be used to enrich provided data with meta-data or in other ways add value to it. In the case of JobTech Dev, SPES provides internally developed functionality as OSS that helps actors to enrich job-ads with meta-data.

- Inspiration and documentation: All four platform providers develop and maintain application examples that can help users to understand how the data can be used. In this sense, the examples can be seen as a source of documentation. However, they may also inspire new use-cases that can be of value both for entrepreneurs and incumbents.

- Development support: All four platform providers also see value in supplying common tools, libraries, and frameworks that can enable actors to create new products and services based on the data.

- $\quad$ Algorithms: The City of Chicago publishes algorithms used within the city, in part for transparency, but also to gain help in making the algorithms more effective and correct. SPES publishes its algorithms for external actors to test and validate the results they produce.

- $\quad$ Products and services: HSL take it one step further and also develop OSS products that can be used both by service providers such as traffic operators and end-users, i.e., the commuters. 
Recommendation: Based on the studied cases, we advise public entities acting as platform providers in an OGD ecosystem to:

- $\quad$ develop and collaborate with the ecosystem's actors on OSS that may improve adoption and innovation based on the data. For example, OSS that enable the collection and enrichment of the data, provide inspiration and documentation, or general support to developers (such as tools, libraries, and infrastructure). The platform provider may also consider sharing their applications that use the data as input, which may include algorithms, as well as tools and consumer-facing products.

\subsection{Data Sharing}

To collaborate on data, the platform providers need to consider what type of data that is (or could be) shared and provided on the platform. If the data is produced externally, the platform provider takes on the role of a broker (Immonen, Palviainen, and Ovaska 2014) or intermediary (Susha et al. 2020), passing data on from the producers to the consumers which is mainly the case for Samtrafiken in the Trafiklab ecosystem. The other three ecosystems provide both internally and externally produced data. Differentiating between organizational structures, as proposed by Oliveira et al. (Oliveira, Lima, and Lóscio 2019), therefore becomes difficult. Instead, to help characterize the types of data shared in an ecosystem, we have distilled four categories of data based on the studied cases; Internally produced data, Actively contributed data, Passively contributed data, and Ecosystem-external data.

Internally produced data originates from within the platform provider. In this case, the platform provider becomes the maintainer of the data and needs to consider how they want actors to be able to contribute to it. In terms of the taxonomy data provided by SPES on the JobTech Dev platform, actors can mainly contribute through the established feedback channels. There are however, examples where actors have contributed data sets directly to SPES which are then incorporated into the data set manually.

Actively contributed data means data that ecosystem actors actively contribute to the platform of, via the tools or infrastructure provided by the platform provider. In this case, the contributing actors maintain their data and can themselves choose when to update what is available on the platform. Examples from the cases studied include the Career-initiative on the JobTech Dev platform, where individuals store their resumé-data, and city bike and car parking data, which is contributed to the HSL DevCom platform.

Passively contributed data means data that the platform provider collects from the ecosystem actors. The original data is maintained by the ecosystem actor, but what is available on the platform is dependent on the platform provider's collection and update procedures. One example is the traffic data from the regional transport authorities that is collected by Samtrafiken. A second example is the job-ads from the job-ad providers that are collected by SPES. A third example is the national data that is collected and broken down on a community level by the City of Chicago.

External ecosystem data refers to data that is maintained in another data ecosystem and collected by the platform provider and made available on the platform, in a direct or enriched way. One example concerns OpenStreetMap, the map data of which, is imported by HSL and used as input to 
their other data sets and API:s. If HSL or any of the ecosystem actors wants to contribute to the map data they can turn directly to the OpenStreetMap project.

Recommendation: Based on the studied cases, we advise public entities acting as platform providers in an OGD ecosystem to:

- facilitate and enable the sharing of data, beyond their own. This can be done either by helping the actors to actively or passively contribute their data. In the active case, the actors use a technical infrastructure or communication channel provisioned by the platform provider. In the passive case, the platform provider collects the data from the actor to then publish it on the platform. A further aspect is to also consider how they can make use of external ecosystems to collaborate on data.

\subsection{Specification and Standardization}

The ecosystem enables a general adoption and promotion of existing standards to promote interoperability among actors but also towards the surrounding or neighboring domains. In the studied cases, standards mentioned have mainly been domain-specific such as the GTFS format referring to traffic data (cf. vertical industry standards (Rudmark 2020)). When the data producers use a different format, they transform it accordingly. Similarly, SPES has chosen to adopt the HRO open standard for their Career-initiative for individuals' resumés to be portable to different recruitment platforms internationally. When there are no existing standards that are suitable, the ecosystem offers a potential venue for collaborating on the development of new ones, as in Trafiklab, that developed a new standard for tickets and payment transactions.

Recommendation: Based on the studied cases, we advise public entities acting as platform providers in an OGD ecosystem to:

- $\quad$ adopt and promote open standards where possible, and to enable interoperability within the ecosystem as well as to the outside. When needed, the platform provider should also take an active role in facilitating the development of new standards to achieve the same goal.

\section{Summary and Conclusions}

OGD ecosystems offer public entities new ways in how they can address their goals and directives. By opening up and enabling collaboration with cross-sectoral actors and the public through an ecosystem, the public entities can establish value networks with the potential to accelerate adoption and innovation based on the data. However, to exploit such benefits of open innovation, these public entities need to move beyond the role of data provider to that of a platform provider that actively enables and facilitates a collaboration on the data and related boundary resources within the ecosystem.

The research goal of this study has been to explore how public entities in the role as platform providers can enable collaboration in OGD ecosystems. To address this goal, we have conducted a 
multiple case study of four OGD ecosystems of varying characteristics in terms of size, domain and geographical coverage. Based on the a cross-case analysis, we synthesize a conceptual model (see Fig. 2) that describe different aspects of OGD ecosystems and how these may vary. These aspects, for example, describes how collaboration can be enabled through different types of ownership of the platform provider, how the ecosystem's scope can vary, what roles the platform provider may undertake, how to enable open collaboration, and how to collaborate in terms of data sharing, OSS development, and standards. For each aspect, we provide recommendations that, along with the model, may provide guidance for public entities acting as platform providers to enable sharing and collaboration on both OGD as well as any boundary resources in the form of OSS and standards.

As this study is limited to exploring the collaboration in four instances of OGD ecosystems, further research is required to validate both the model and recommendations, create a deeper understanding, and improve the external validity (Runeson et al. 2012). Readers should consider the context of the platform providers and their ecosystems as reported, and adopt an analytical generalization to cases with similar characteristics (Runeson et al. 2012).

In future research, we intend to continue our exploration into the collaborative aspects of OGD ecosystems in terms of both data, OSS, and standards, taking into account both the platform providers' and ecosystem actors' perspectives. We further aim to theorize how the data and software can be considered as commons and what role public entities can play in preserving sustainable provisioning and the development of them.

\section{References}

Alves, Carina, Joyce Oliveira, and Slinger Jansen. 2018. “Understanding Governance Mechanisms and Health in Software Ecosystems: A Systematic Literature Review." In Enterprise Information Systems, edited by Slimane Hammoudi, Michał Śmiałek, Olivier Camp, and Joaquim Filipe, 517-42. Cham: Springer International Publishing.

Attard, Judie, Fabrizio Orlandi, Simon Scerri, and Sören Auer. 2015. "A Systematic Review of Open Government Data Initiatives." Government Information Quarterly 32 (4): 399-418.

Baars, Alfred, and Slinger Jansen. 2012. “A Framework for Software Ecosystem Governance." In Software Business, edited by Michael A. Cusumano, Bala Iyer, and N. Venkatraman, 168-80. Berlin, Heidelberg: Springer Berlin Heidelberg.

Coyle, Diane, Stephanie Diepeveen, and Julia Wdowin. 2020. “The Value of Data Summary Report." The Bennett Institute, Cambridge.

Dal Bianco, Vittorio, Varvana Myllärniemi, Marko Komssi, and Mikko Raatikainen. 2014. “The Role of Platform Boundary Resources in Software Ecosystems: A Case Study." In 2014 Ieee/Ifip Conference on Software Architecture, 11-20. IEEE.

Dawes, Sharon S, Lyudmila Vidiasova, and Olga Parkhimovich. 2016. "Planning and Designing Open Government Data Programs: An Ecosystem Approach." GIQ 33 (1): 15-27. 
De Noni, Ivan, Andrea Ganzaroli, and Luigi Orsi. 2013. "The evolution of OSS governance: A dimensional comparative analysis." SJM 29 (3): 247-63.

Ernst, Neil, and Gail C. Murphy. 2012. "Case Studies in Just-in-Time Requirements Analysis.” In 2nd International Workshop on Empirical Requirements Engineering, 25-32. EmpiRE'12. Chicago, IL, USA: IEEE. https:// doi.org/10.1109/EmpiRE.2012.6347678.

Feller, Joseph, Brian Fitzgerald, and others. 2002. Understanding Open Source Software Development. Addison-Wesley London.

Franco-Bedoya, Oscar, David Ameller, Dolors Costal, and Xavier Franch. 2017. “Open source software ecosystems: A Systematic mapping." IST 91: 160-85.

Gao, Yingying, and Marijn Janssen. 2020. “Generating Value from Government Data Using Ai: An Exploratory Study." In Electronic Government, edited by Gabriela Viale Pereira, Marijn Janssen, Habin Lee, Ida Lindgren, Manuel Pedro Rodríguez Bolívar, Hans Jochen Scholl, and Anneke Zuiderwijk, 319-31. Cham: Springer International Publishing.

Gawer, Annabelle, and Michael A. Cusumano. 2002. Platform Leadership: How Intel, Microsoft, and Cisco Drive Industry Innovation. Vol. 5. Boston, MA.: Harvard Business School Press.

Ghazawneh, Ahmad, and Ola Henfridsson. 2013. "Balancing Platform Control and External Contribution in Third-Party Development: The Boundary Resources Model." Information Systems Journal 23 (2): 173-92.

Harrison, Teresa M, Theresa A Pardo, and Meghan Cook. 2012. "Creating Open Government Ecosystems: A Research and Development Agenda." Future Internet 4 (4): 900-928.

Iansiti, Marco, and Roy Levien. 2004. The Keystone Advantage: What the New Dynamics of Business Ecosystems Mean for Strategy, Innovation, and Sustainability. HBP.

Immonen, Anne, Marko Palviainen, and Eila Ovaska. 2014. "Requirements of an Open Data Based Business Ecosystem." IEEE Access 2: 88-103.

Jansen, Slinger. 2014. "Measuring the Health of Open Source Software Ecosystems: Beyond the Scope of Project Health." Inf. And Software Technology 56 (11): 1508-19.

Jansen, Slinger. 2020. “A Focus Area Maturity Model for Software Ecosystem Governance." Information and Software Technology 118.

Jansen, Slinger, Anthony Finkelstein, and Sjaak Brinkkemper. 2009. “A Sense of Community: A Research Agenda for Software Ecosystems." In 31st International Conference on Software Engineering Companion Volume, 187-90. ICSE'09. Vancouver, BC, Canada: IEEE. https://doi.org/10.1109/ICSECOMPANION.2009.5070978.

Janssen, Marijn, Yannis Charalabidis, and Anneke Zuiderwijk. 2012. "Benefits, Adoption Barriers and Myths of Open Data and Open Government." Information Systems Mgmt 29 (4): 258-68. 
Kitsios, Fotis, Nikolaos Papachristos, and Maria Kamariotou. 2017. "Business Models for Open Data Ecosystem: Challenges and Motivations for Entrepreneurship and Innovation." In 19th Conference on Business Informatics, 1:398-407. IEEE.

Lakomaa, E., and J. Kallberg. 2013. “Open Data as a Foundation for Innovation: The Enabling Effect of Free Public Sector Information for Entrepreneurs." IEEE Access 1: 558-63.

Linåker, Johan, and Per Runeson. 2020a. "Collaboration in Open Government Data Ecosystems: Open Cross-Sector Sharing and Co-Development of Data and Software." In Electronic Government, edited by Gabriela Viale Pereira, Marijn Janssen, Habin Lee, Ida Lindgren, Manuel Pedro Rodríguez Bolívar, Hans Jochen Scholl, and Anneke Zuiderwijk, 290-303. Cham: Springer International Publishing.

Linåker, Johan, and Per Runeson. 2020b. "Public Sector Platforms Going Open: Creating and Growing an Ecosystem with Open Collaborative Development." In Proceedings of the 16th International Symposium on Open Collaboration, 1-10.

Lindman, Juho, Tomi Kinnari, and Matti Rossi. 2015. “Business Roles in the Emerging Open-Data Ecosystem." IEEE Software 33 (5): 54-59.

Mhamdia, Amel Ben Hadj Salem. 2013. "Performance Measurement Practices in Software Ecosystem." Int. Journal of Productivity and Performance Management 62 (5): 514-33.

Munappy, A., J. Bosch, H. H. Olsson, A. Arpteg, and B. Brinne. 2019. “Data Management Challenges for Deep Learning." In 2019 45th Euromicro Conference on Software Engineering and Advanced Applications (Seaa), 140-47. https:// doi.org/10.1109/SEAA.2019.00030.

Munir, Hussan, Krzysztof Wnuk, and Per Runeson. 2016. “Open Innovation in Software Engineering: A Systematic Mapping Study." Empirical Software Engineering 21 (2): 684-723.

Najafabadi, M. Mahdi, and Luis Luna-Reyes. 2017. “Open Government Data Ecosystems: A Closed-Loop Perspective." In Proc. 50th Hicss.

Nakakoji, Kumiyo, Yasuhiro Yamamoto, Yoshiyuki Nishinaka, Kouichi Kishida, and Yunwen Ye. 2002. "Evolution Patterns of Open-Source Software Systems and Communities." In Proc. Int. Workshop on Principles of Software Evolution, 76-85. Orlando, Florida: ACM.

Oliveira, Marcelo Iury S, Glória de Fátima Barros Lima, and Bernadette Farias Lóscio. 2019. “Investigations into Data Ecosystems: A Systematic Mapping Study." Knowledge and Information Systems, $1-42$.

Riehle, Dirk. 2012. “The Single-Vendor Commercial Open Course Business Model.” Information Systems and E-Business Management 10 (1): 5-17.

Robles, Gregorio, Jonas Gamalielsson, and Björn Lundell. 2019. “Setting up Government 3.0 Solutions Based on Open Source Software: The Case of X-Road." In International Conference on Electronic Government, 69-81. Springer. 
Rudmark, Daniel. 2020. “Open Data Standards: Vertical Industry Standards to Unlock Digital Ecosystems." In 53rd Hawaii International Conference on System Sciences.

Rudmark, Daniel, and Anders Hjalmarsson-Jordanius. 2019. "Harnessing Digital Ecosystems Through Open Data-Diagnosing the Swedish Public Transport Industry." In European Conference of Information Systems (Ecis).

Runeson, Per. 2019. “Open Collaborative Data - Using OSS Principles to Share Data in SW Engineering." In 41st Acm/leee Int. Conf. On Software Eng. (ICSE): New Ideas and Emerging Results (Nier) Track, 25-28. Montreal, Canada.

Runeson, Per, Martin Höst, Austen Rainer, and Björn Regnell. 2012. Case Study Research in Software Engineering - Guidelines and Examples. Wiley.

Safarov, Igbal, Albert Meijer, and Stephan Grimmelikhuijsen. 2017. "Utilization of Open Government Data: A Systematic Literature Review of Types, Conditions, Effects and Users." Information Polity 22 (1): 1-24.

Sieber, Renee E, and Peter A Johnson. 2015. “Civic Open Data at a Crossroads: Dominant Models and Current Challenges." Government Information Quarterly 32 (3): 308-15.

Susha, Iryna, Maartje Flipsen, Wirawan Agahari, and Mark de Reuver. 2020. “Towards Generic Business Models of Intermediaries in Data Collaboratives: From Gatekeeping to Data Control." In Electronic Government, edited by Gabriela Viale Pereira, Marijn Janssen, Habin Lee, Ida Lindgren, Manuel Pedro Rodríguez Bolívar, Hans Jochen Scholl, and Anneke Zuiderwijk, 304-15. Cham: Springer International Publishing.

Zuiderwijk, Anneke, Marijn Janssen, and Chris Davis. 2014. “Innovation with Open Data: Essential Elements of Open Data Ecosystems." Information Polity 19 (1, 2): 17-33.

\section{About the Authors}

\section{Johan Linåker}

Dr. Johan Linåker is a postdoctoral researcher at Lund University and a member of its Software Engineering Research Group. His research interests include empirical software engineering research in industry and public sector in the context of open innovation. He is specifically interested in the areas of Open Source Software and Open Data, as well as software requirements engineering and product management.

\section{Per Runeson}

Dr. Per Runeson is a professor of software engineering at Lund University, Sweden, and leader of its Software Engineering Research Group (SERG). His research interests include empirical research in industry on software development and management methods. He is particularly interested in studies on testing, and Open Source Software and Open Data strategies. He is the principal author of "Case study research in software engineering", and has served as general chair for ESEM, program chair for ICST, ISSRE, and TAIC PART, special issue editor for EMSE, STVR, and SQJ, being PC member for 30+ conferences, and serves currently on the editorial board of the EMSE and STVR journals. 